\title{
Estudo retrospectivo dos achados cardiorrespiratórios e laboratoriais de cães atendidos para avaliação pré-operatória em um serviço de cardiologia e doenças respiratórias (2017-2021)*
}

\author{
Retrospective study of cardiorespiratory and laboratory findings \\ in dogs treated for preoperative evaluation in a cardiology and $r$ \\ espiratory disease service (2017-2021)
}

\author{
Eduarda de Oliveira Silva Lima Machado, ${ }^{* *}$ Bruna Pereira Gonçalves, ${ }^{* *}$ \\ Jaíne da Silva, ${ }^{* *}$ Mário dos Santos Filho***
}

\begin{abstract}
Resumo
Os cães idosos são acometidos com frequência por enfermidades no sistema cardiovascular e respiratório, estando muita das vezes assintomáticos até terem indicação para algum procedimento cirúrgico que requer a avaliação pré-operatória, momento este que muitos achados clínicos poderão ser detectados, ao ponto da necessidade de tratamento prévio à intervenção cirúrgica. Diante disso, o trabalho tem por objetivo detectar as principais manifestações cardiorrespiratórias e sua correlação aos dados encontrados nos exames complementares e informações epidemiológicas de 138 cães atendidos em um serviço especializado em cardiologia e doenças respiratórias, no período de 2017 a 2021, para fins de risco cirúrgico. Foi possível observar maior predominância de animais idosos, de pequeno porte e do sexo feminino. Dentre as doenças cardíacas, a doença degenerativa crônica de mitral foi a mais prevalente, seguida da dirofilariose. Em relação as afecções respiratórias, a bronquite crônica e o colapso de traqueia foram mais diagnosticadas, seguidas da broncopneumonia e da síndrome braquicefálica. Quando da observação do ritmo cardíaco por meio do eletrocardiograma, a arritmia sinusal, ritmo sinusal e sinus arrest foram os principais achados. Quanto aos achados laboratoriais, foi possível observar aumento da média de hematimetria, redução da média do hematócrito e das plaquetas. Entretanto, alguns animais apresentaram azotemia, leucocitose e eosinofilia. Observou-se que pacientes com doenças cardíacas em estágios de congestão possuem redução da pressão arterial, aumento da hematimetria e taquiarritmias, como taquicardia sinusal e/ou complexos atriais prematuros. Pacientes com idade mais avançada tendem a ter redução dos valores de leucometria global e maior probabilidade de manifestação de aumento da pressão arterial. Portadores de dirofilariose cursam com aumento de eosinófilos.
\end{abstract}

Palavras-chave: afecções respiratórias, cardiopatias, idoso, medicina preventiva, risco cirúrgico.

\begin{abstract}
Elderly dogs are frequently affected by diseases in the cardiovascular and respiratory system, being often asymptomatic until they are indicated for a surgical procedure that requires preoperative evaluation, at which point many clinical findings can be detected, to the point of need for treatment prior to surgical intervention. Therefore, the work aims to detect the main cardiorespiratory manifestations and their correlation to data found in complementary exams and demographic information of 138 animals treated at a service specialized in cardiology and respiratory diseases, from 2017 to 2021 , for risk purposes surgical. It was possible to observe a greater predominance of elderly, small and female animals. Among heart diseases, chronic degenerative mitral disease was the most prevalent, followed by heartworm disease. Regarding respiratory affections, chronic bronchitis and tracheal collapse were more commonly diagnosed, followed by bronchopneumonia and brachycephalic syndrome. When observing the cardiac rhythm through the electrocardiogram, sinus arrhythmia, sinus rhythm and sinus arrest were the main findings. As for laboratory findings, it was possible to observe an increase in mean hematimetry, a reduction in mean hematocrit and platelets. However, some animals showed azotemia, leukocytosis and eosinophilia. It was observed that patients with cardiac diseases in stages of congestion have reduced blood pressure, increased hematimetry and tachyarrhythmias, such as sinus tachycardia and/or premature atrial complexes. Older patients tend to have reduced global white blood cell values and more likely to manifest increased blood pressure. Heartworm patients have an increase in eosinophils.
\end{abstract}

Keywords: respiratory disorders, heart disease, elderly, preventive medicine, surgical risk.

\footnotetext{
${ }^{*}$ Recebido em 29 de agosto de 2021 e aceito em 11 de novembro de 2021.

**Universidade de Vassouras, Curso de Medicina Veterinária, Vassouras, RJ, Brasil.

***Universidade de Vassouras, Curso de Medicina Veterinária, Vassouras, RJ, Brasil \& Universidade Federal Rural do Rio de Janeiro, Programa de PósGraduação em Medicina Veterinária - Patologia e Ciências Clínicas, Seropédica, RJ, Brasil.
} 


\section{Introdução}

A compreensão das alterações clínicas apresentadas por cães é essencial para o clínico de pequenos animais, contribuindo principalmente na formulação de diagnósticos diferenciais. A detecção de sinais clínicos ou diagnóstico de doenças podem ocorrer durante exame anual de rotina, durante a avaliação de um paciente para uma determinada afecção focada em outros sistemas específicos, ou durante o exame pré-operatório. $\mathrm{O}$ exame pré-operatório permite a identificação de possíveis enfermidades existentes, mesmo nos casos assintomáticos, o que é essencial para estabilização clínica do paciente, a fim de prepará-lo para o procedimento cirúrgico e, assim, reduzir riscos de complicações trans-operatórias e pós-operatórias (CASTRO et al. 2009).

Os cães são acometidos com frequência por enfermidades no sistema cardiovascular e respiratório, podendo não manifestarem sintomas até serem submetidos às intervenções anestésicas (SANTO et al. 2013). Dentre as cardiopatias mais frequentemente diagnosticadas em cães de raça de pequeno porte, está a doença valvar degenerativa crônica de mitral (DVDCM), enquanto que nas de grande porte, a cardiomiopatia dilatada (CMD). Independentemente de sua causa, a principal consequência de uma cardiopatia vem a ser a insuficiência cardíaca congestiva, foco da conduta terapêutica (KEENE et al., 2019). Em relação as afecções respiratórias, a bronquite crônica canina vem a ser uma das mais comumente diagnosticadas em cães (SANTOS et al. 2013).

Segundo Carvalho et al. (2009), exames pré-operatórios devem ser realizados, principalmente em animais acima de 6 anos, devido à maior predisposição de enfermidades cardiorrespiratórias. Além da anamnese e exame físico, são necessários a realização de exames como eletrocardiograma (ECG), radiografia torácica, ecocardiograma (ECO) e avaliação laboratorial hematológica. Mediante a interpretação dos resultados dos exames complementares, o médico veterinário decidirá se o animal precisará de protocolo terapêutico para ser estabilizado ou seguirá para o procedimento cirúrgico.

Nesse contexto, sabendo da importância da avaliação préoperatória, o presente estudo visa detectar as principais manifestações cardiorrespiratórias, bem como correlacionar os dados encontrados nos exames clínicos e complementares com os dados epidemiológicos dos animais atendidos para o risco cirúrgico em um serviço especializado em cardiologia e doenças respiratórias, no período entre março de 2017 a março de 2021.

\section{Material e Métodos}

Foram compilados dados de prontuários médicos de cães atendidos em um serviço especializado em cardiologia e doenças respiratórias, no período entre março de 2017 a março de 2021.

Das fichas clínicas avaliadas, foram recolhidos os dados demográficos, dados relativos aos exames clínicos e complementares realizados no pré-cirúrgico de procedimentos eletivos ou não, como hemograma, bioquímica sérica, aferição de pressão arterial, eletrocardiograma e ecodopplercardiograma. No hemograma foram coletadas as alterações do eritrograma, leucograma, plaquetograma e proteína plasmática total (PPT).
$\mathrm{Na}$ bioquímica sanguínea foram processados os exames bioquímicos com dosagens de ureia e creatinina, alanina aminotransferase (ALT), fosfatase alcalina (FA) e albumina.

Foram incluídos no estudo somente os animais cujos dados acima descritos estivessem completos, perfazendo o total de 138 prontuários, entre 224 compilados no presente período.

Os dados foram planilhados em aplicativo de editor de tabelas (Microsoft Excel ${ }^{\odot}$ ), e submetidos a estatística por meio do Software Bioestat ${ }^{\circledR}$ 5.0. Para os achados hematológicos e bioquímicos foi realizado estatística descritiva, onde os dados foram expressos na forma de média e desvio padrão, bem como os valores mínimos e os valores máximos. Para análise estatística dos dados demográficos foi realizada também a estatística descritiva, em valores percentuais e absolutos, bem como média e desvio padrão dos valores quantitativos.

O teste qui-quadrado e a correlação de Pearson foram utilizados para comparação entre os dados dos exames clínicos e complementares, obedecendo ainda, significância de 0,05 e p-valor de até 0,05 para consideração de hipótese nula.

\section{Resultados}

Após a triagem inicial das fichas clínicas, foram selecionados 138 cães para compor a amostra do estudo. Dentre os animais que passaram pelo risco cirúrgico, $35 \%$ eram portadores de neoplasia mamária; $18 \%$ de afecções dentárias; $13 \%$ de doenças ortopédicas; $11 \%$ de afecções da cavidade abdominal; e $9 \%$ realizariam cirurgias eletivas como orquiectomia e ovariossalpingohisterectomia (Tabela 1).

Tabela 1: Motivo da realização da avaliação pré-operatória de cães pelo setor de cardiologia e doenças respiratórias.

Motivo da realização da avaliação pré-operatória

\begin{tabular}{ll}
\hline Total de animais $(\mathrm{n})$ & $\mathrm{n}=138$ \\
Afecções & Neoplasia mamária $-48 / 128(35 \%)$ \\
& Afeç̧ões dentárias - 25/138 (18\%) \\
& Doenças ortopédicas - 18/138 (13\%) \\
& $\begin{array}{l}\text { Afecções da cavidade abdominal* - 15/138 } \\
(11 \%)\end{array}$ \\
& Castração - 12/138 (9\%) \\
& Outros tumores - 10/138 (7\%) \\
& Outras $^{* *}-10 / 138(7 \%)$
\end{tabular}

*laparotomia exploratória, esplenectomia, hérnias.

**amputações, torção de lobos pulmonares, doenças oftalmológicas, piometra, nefrectomia, cálculos vesicais, cesarianas.

A média de idade dos animais atendidos foi de 102 \pm 18 meses, sendo $60 \%$ fêmeas e $40 \%$ machos. Aproximadamente $15 \%$ dos animais ( $n=20$ animais), eram cães sem raça definida, seguido de Poodle ( $n=16$ animais) e Shih-tzu ( $n=14$ animais). Yorkshire, Maltês e Pug vem em seguida, com mesmos valores absolutos ( $\mathrm{n}$ = 12 animais cada). Quanto ao peso, a média dos animais foi de $7,88 \pm 5,86 \mathrm{Kg}$. Os dados estão detalhados na tabela 2 a seguir. 
Tabela 2: Dados epidemiológicos dos cães atendidos pelo setor de cardiologia e doenças respiratórias, para fins de avaliação pré-operatória.

\begin{tabular}{ll}
\hline \multicolumn{2}{c}{ Dados demográficos dos cães atendidos pelo setor } \\
de cardiologia
\end{tabular}

Ao exame clínico, 53 animais apresentaram algum grau de sopro à ausculta, contudo sem alteração hemodinâmica, descartada pelo exame ecodopplercardiográfico, incluídos, segundo consenso ACVIM (KENNE et al., 2019), para doença valvar degenerativa crônica de mitral (DVDCM), no grupo B1. 25 animais apresentavam-se com sopro, e ao ecodopplercardiograma, apresentaram alterações hemodinâmicas, sendo estes 15 animais no estágio $B 2$ e 10 animais no grupo $C$, já em processo congestivo.
A média dos valores de pressão arterial, foi de $130 \pm 19 \mathrm{mmHg}$. Houve correlação positiva forte entre a idade do paciente com a pressão arterial $(0,812, p=0,011,0,05 \%)$. Entretanto, quando comparados peso com pressão arterial $(0,180, p=0,461,0,05 \%)$ e peso com a idade $(0,215, p=0,389,0,05 \%)$, a correlação foi desprezível.

A doença respiratória mais prevalente foi a bronquite crônica ( $n=$ 56 animais), seguida do colapso de traquéia ( $n=25$ animais), boncopneumonia ( $n=18$ animais) e, por fim a síndrome braquicefálica ( $n=9$ animais). Quanto a doença cardíaca, cerca de $20 \%$ ( $n=$ 27/138) dos animais apresentavam insuficiência cardíaca congestiva (ICC), tendo a doença valvar crônica de mitral a doença mais prevalente. 15 animais eram portadores de dirofilariose. Dentre os animais em ICC, houve moderada correlação negativa com os valores de pressão arterial $(-0,645, p=0,435,0,05 \%)$.

Quanto aos achados eletrocardiográficos, arritmia sinusal foi o achado mais frequente ( $n=75$ animais), seguido do ritmo sinusal $(n=27$ animais), Sinus arrest $(n=17$ animais), Bloqueio Átrioventricular de Primeiro Grau (BAV 1) ( $n=13$ animais), taquicardia sinusal ( $n=10$ animais), Complexo Atrial Prematuro (CAPS) $(n=9$ animais), Bloqueio Átrio-Ventricular de Segundo Grau (BAV 2) (n= 3 animais) e taquicardia ventricular ( $n=1$ animal). Considerando somente os animais com alteração cardíaca em congestão, 20 possuíram algum grau de taquiarritmia (Taquicardia sinusal e/ ou CAP), perfazendo correlação fortemente positiva $(0,789$, $\mathrm{p}=0,356,0,05 \%)$.

No que diz respeito as alterações laboratoriais relacionadas ao hemograma, pode-se constatar que os valores médios de hematimetria se encontram acima dos valores de referência, enquanto os valores do hematócrito e das plaquetas encontramse abaixo (Jain, 1993). Quanto os exames de bioquímica avaliados e seus respectivos resultados pode-se observar todos os valores médios dentro dos valores de referência, apesar da ureia, ALT, AST e FA apresentarem valores máximos muito altos (Kaneko et al., 1997). Os dados dos exames hematológicos e bioquímicos da população estudada, estão apresentados resumidamente, nas tabelas abaixo (Tabela 2 e Tabela 3).

Tabela 3: Achados hematológicos dos pacientes caninos representados por média $(\mathrm{X})$ e o desvio padrão (DP), valores máximos e mínimos, seguido dos valores de referência.

\begin{tabular}{ccccc}
\hline Hemograma & X \pm DP & Máximo & Mínimo & Valores de Referência \\
\hline Hematimetria & $9,34 \pm 1,62$ & 12,00 & 6,00 & $5-8,5 \times 10^{6} / \mathrm{mm}^{3}$ \\
Hematócrito & $32,37 \pm 7,21$ & 50,00 & 24,00 & $37-55 \%$ \\
Hemoglobina & $12,55 \pm 2,83$ & 17,00 & 9,00 & $12-18 \mathrm{~g} / \mathrm{dL}$ \\
VCM & $64,30 \pm 8,31$ & 77,00 & 45,00 & $60-77 \mathrm{fl}$ \\
CHCM & $32,84 \pm 3,01$ & 37,00 & 27,00 & $32-36 \%$ \\
Leucometria & $8956,52 \pm 1789,54$ & 12000,00 & 5000,00 & $6-17 \times 10^{6} / \mathrm{mm}^{3}$ \\
Basófilo & $0,00 \pm 0,00$ & 0,00 & 0,00 & $\mathrm{Raros}$ \\
Eosinófilo & $535,14 \pm 253,90$ & 1000,00 & 150,00 & $100-750 / \mathrm{mm}^{3}$ \\
Bastão & $78,98 \pm 101,59$ & 250,00 & 0,00 & $0-300 / \mathrm{mm}^{3}$ \\
Segmentado & $9521,73 \pm 1268,46$ & 12000,00 & 7000,00 & $3.000-11.400 / \mathrm{mm}^{3}$ \\
Linfócito & $2829,71 \pm 1283,66$ & 5000,00 & 750,00 & $1.000-4.800 / \mathrm{mm}^{3}$ \\
Monócito & $928,98 \pm 387,05$ & 14000,00 & 150,00 & $150-1.350 / \mathrm{mm}^{3}$ \\
Plaquetas & $197,10 \pm 44,70$ & 300,00 & 150,00 & $200.000-500.000 / \mathrm{mm}^{3}$ \\
PPT & $6,73 \pm 0,99$ & 8,00 & 5,00 & $6-7,5 \mathrm{~g} / \mathrm{dL}$ \\
\hline
\end{tabular}

VCM: Volume Corpuscular Médio; CHCM: Concentração de Hemoglobina Corpuscular Média. 
Tabela 4: Achados bioquímicos dos pacientes caninos representados por média (X) e o desvio padrão (DP), valores máximos e mínimos, seguido dos valores de referência.

\begin{tabular}{ccccc}
\hline Bioquímica & $\mathbf{X} \pm \mathrm{DP}$ & Máximo & Mínimo & Valores de Referência \\
\hline Ureia & $30,32 \pm 14,15$ & 60,00 & 15,00 & $15-40 \mathrm{mg} / \mathrm{dL}$ \\
Creatinina & $0,88 \pm 0,87$ & 3,00 & 0,00 & $0,5-1,7 \mathrm{mg} / \mathrm{dL}$ \\
ALT & $61,67 \pm 33,46$ & 150,00 & 15,00 & $10-109 \mathrm{U} / \mathrm{L}$ \\
AST & $74,17 \pm 45,54$ & 200,00 & 15,00 & $10-88 \mathrm{U} / \mathrm{L}$ \\
FA & $69,85 \pm 52,36$ & 200,00 & 25,00 & $1-114 \mathrm{U} / \mathrm{L}$ \\
Albumina & $2,28 \pm 0,72$ & 3,00 & 1,00 & $2,3-3,1 \mathrm{~g} / \mathrm{dL}$ \\
\hline
\end{tabular}

ALT: alanina aminotransferase; AST: aspartato aminotransferase; FA: fosfatase alcalina.

Correlacionando a idade dos pacientes, com os níveis séricos de ureia e creatinina, houve correlação positiva (respectivamente, $0,893, p=0,057,0,05 \%$ e 0,972, $p=0,042,0,05 \%$ ). Quanto a comparação da pressão arterial com creatinina e ureia, houve correlação positiva com os níveis de creatinina $(0,805, p=0,125$, 0,05\%), contudo sem correlação com ureia.

Comparando os animais com doença cardíaca e sem doença cardíaca, notou-se correlação do primeiro com os níveis de hemácias $(0,732, p=0,365,0,05 \%)$, enquanto não houve correlação dos não-cardiopatas com essa alteração. Outra análise observada, foi a dos pacientes portadores de ICC correlacionandose com proteínas plasmáticas $(0,604, p=0,452,0,05 \%)$.

Comparando-se a idade, com a leucometria, observou-se correlação negativa $(-0,689, p=0,739,0,05 \%)$. Entre os portadores de dirofilariose, observou-se correlação positiva com a eosinofilia $(0,856, p=0,789,0,05 \%)$.

$\mathrm{Na}$ comparação do percentual de hematócrito com os valores de creatinina, observou-se correlação positiva $(0,337, p=0,246$, 0,05\%). Comparando-se ALT, AST, FA e Albumina, houve correlação entre ALT e Albumina (0,992, $p=0,038,0,05 \%)$, entre AST e FA $(0,654, p=0,453,0,05 \%)$ e AST e albumina $(0,689$, $p=0,356,0,05 \%)$.

\section{Discussão}

O exame pré-operatório é essencial para avaliar o risco de problemas durante todo o período transoperatório, bem como observar alterações que podem ser limitantes e devem ser tratadas antes de qualquer procedimento. Deve ser feito principalmente em animais com mais de seis anos devido à maior predisposição de doenças cardíorespiratórias e para a escolha de quais fármacos podem ser usados no ato anestésico (CARVALHO, et al., 2009). No presente estudo, a maioria dos cães eram pacientes oncológicos, principalmente decorrente da neoplasia mamária, que para seu estadiamento necessitouse de todos os exames de rotina. Enquanto outras avaliações foram para procedimentos odontológicos e ortopédicos, de modo geral, demonstrando e justificando a casuística relacionada com a idade e, por consequência, a necessidade de realização do risco pré-operatório.

A média de idade dos animais apresentou-se alta e com pouca margem de desvio padrão, sendo possível observar maior prevalência de animais idosos. Dado que vai de encontro com os achados por Rocha et al. (2013), que observaram que cães na faixa etária entre oito e dez anos comumente apresentam problemas cardiorrespiratórios e, mais do que isso, necessitam avaliação pré-operatória em cirurgias eletivas ou urgente. Vale ressaltar que, do ato da avaliação, nos casos de emergências cirúrgicas, como a piometra, independente do risco préoperatório, a necessidade de seu procedimento se sobrepõe sob qualquer tratamento prévio, sendo alguns dos pacientes atendidos incluídos nessa triagem, possivelmente os mesmos que obtiveram valores clínicos e laboratoriais com significativa alteração.

Em relação ao sexo dos animais, foi observado maior predomínio das fêmeas se comparados a machos. Esta informação pode estar associada ao fato dos tipos de procedimentos indicados, uma vez que se observou grande incidência de exames préoperatórios para mastectomia e piometra. Estes dados vão de encontro ao reportado por Ataide et al. (2020) que observou percentual ou valores próximos do presente estudo, em termos de proporcionalidade.

O peso médio dos animais do estudo foi relativamente baixo, que denota uma relação com o porte dos animais atendidos. Essa relação é reforçada quando observado a ampla distribuição das raças, principalmente as de pequeno porte. Isso pode estar relacionado com o perfil dos tutores que estão buscando cada vez mais animais com menor tamanho, mais sociáveis e com menor ocupação de espaço, pela verticalização dos grandes centros, configurando uma mudança do perfil dos pets nos últimos anos (DALMAS, 2019).

Dentre os animais que apresentaram sopro à ausculta e, quando realizado o exame ecodopplercardiográfico não apresentaram alteração hemodinâmica, foram classificados em DVDCM estágio B1 (KEENE et al., 2019). Esse achado pode estar relacionado com a progressão da doença, que de uma maneira geral apresenta início lento dos sinais clínicos (Borgarelli et al. 2012; Keene et al. 2019). Normalmente, a maioria dos cães apresentam início de um sopro reconhecível de regurgitação da válvula mitral, com manutenção do débito cardíaco. De acordo com Haggstrom et al. (2010) e Rasmussen et al. (2012), as arritmias sinusais são frequentemente encontradas nos estágios iniciais da doença valvar, fato que pode justificar os achados eletrocardiográficos do estudo, visto que nesse estágio ainda não há remodelamento cardíaco, tal qual poderia desarranjar o sistema de condução com geração de pontos ectópicos de estímulos (SANTILLI et al., 2020).

Dentre os animais que apresentaram sopro e, ao ecodopplercardiograma demonstraram alterações hemodinâmicas, 15 animais foram classificados no estágio B2 e 10 animais no estágio C, 
manifestando a doença valvar crônica como a principal causa de ICC no estudo. À medida que a doença valvar progride, há redução do débito cardíaco e propensão na diminuição da pressão arterial (TILLEY; BURTINICK, 2004). Diante disso, mecanismos compensatórios neuro-humorais são ativados e a fase compensatória permite que a maioria dos cães permaneçam assintomáticos por um período prolongado, como visto nos pacientes em estágio B2. Entretanto, a ativação do tônus simpático para manutenção do débito cardíaco, culmina em vasoconstrição periférica e taquicardia (RASMUSSEn et al., 2012), podendo justificar os achados eletrocardiográficos do presente estudo, que demonstraram elevada frequência de taquicardia sinusal, principalmente observando individualmente os pacientes neste estágio da doença cardíaca.

Uma característica comum de todas as repostas compensatórias é que, em curto prazo, seus efeitos são benéficos, porém, em longo prazo, são deletérios, como visto na exposição crônica a altos níveis de catecolaminas. A persistência do tônus simpático promove um maior requerimento de oxigênio por parte do miocárdio e remodelamento cardíaco devido a congestão instalada (KITTLESON, 2005), informação que pode justificar a correlação positiva entre 20 pacientes em congestão (estágio C) e a presença de taquiarritmias, como taquicardia sinusal e/ou complexos atriais prematuros (CAPs), nos achados eletrocardiográficos deste estudo. Aliado a isso, pode-se observar que quanto mais grave for a evolução da doença, menor poderá ser a pressão arterial, visto que, o processo congestivo instalado nestes pacientes podem culminar em ineficiência da manutenção do débito mesmo diante do aumento da frequência cardíaca, uma vez que o volume sistólico final está muito reduzido, perfazendo o momento de queda de pressão (a lembrar, Débito Cardíaco = VS (Volume Sistólico) $\mathrm{x}$ FC (Frequência Cardíaca). Estes dados justificam a moderada correlação negativa entre a pressão arterial e os animais em insuficiência cardíaca congestiva.

As doenças associadas com o aparecimento da hipertensão arterial sistêmica são mais frequentes em animais geriátricos. Isso se deve ao fato de animais com idade mais avançada estarem mais propensos as doenças de curso crônico, como doenças metabólicas e doenças do sistema renal, sendo reforçada ao fato da relação da idade se somar, também, com os níveis séricos das bioquímicas renais (METZGER, 2005). Ainda, a ICC por si só justificaria a azotemia pré-renal estabelecida na síndrome cardiorrenal, com aumento dos níveis séricos das mesmas enzimas (NICOLLE et al. 2007), justificando a correlação entre a idade do paciente com a pressão arterial.

Foi possível observar uma correlação positiva entre pressão arterial com os níveis séricos de creatinina. Isso se deve ao fato de que este produto é exclusivamente eliminado pelos rins e, quando a pressão arterial se encontra descompensada, o organismo tende a compensá-la ativando mecanismos neurohumorais, os quais originam alterações hemodinâmicas que diminuem a taxa de filtração glomerular e consequentemente a eliminação da creatinina, a qual se concentra em níveis elevados na circulação sanguínea (MEUTEUN, 2015). Quando comparada pressão arterial com os níveis séricos de ureia, não houve correlação, e isto pode ser justificado pelo fato de a ureia não ser um marcador renal específico, pois existem fatores extra-renais que influenciam no seu teor sanguíneo, tais quais a diminuição da metabolização de amônia pelo tecido hepático, principalmente nos pacientes idosos, altamente prevalentes no estudo (LOPES; VEIGA, 2008).

Ainda, dentre as doenças cardíacas, 15 animais demonstraram ser portadores de dirofilariose. $O$ fato dos animais do presente estudo possuírem idade mais avançada poderia justificar a incidência desta doença parasitária. De acordo com os dados encontrados por Almeida et al. (2001), foi possível observar maiores percentuais de amostras positivas no intervalo de idade entre oito e dez anos, mostrando uma maior frequência do parasitismo com o aumento da idade.

Ademais, segundo Almeida et al. (2001), o parasitismo por Dirofilaria immitis, pode estar relacionado com o porte do animal. Estudos mais antigos demostraram maior frequência de animais de grande porte portadores de dirofilariose, com a justificativa de que possuíam atividades de guarda, caça ou pastoreio, sendo desta forma, mais expostos aos mosquitos hospedeiros da microfilária, aumentando assim suas chances de infecção, ao contrário das raças de pequeno porte criadas, comumente, em ambientes fechados. No entanto, com as mudanças climáticas, percebe-se uma alteração no panorama epidemiológico, principalmente por climas mais quentes e úmidos favorecerem a formação de seus vetores em ambientes urbanos, influenciando assim no aumento dos casos de animais de pequeno porte, mesmo distante de regiões tidas como endêmicas, como o encontrado neste trabalho (FILHO et al., 2021). Além disso, o perfil dos atendimentos onde os dados foram coletados, se baseiam em animais cosmopolitas, advindos de diversas regiões, entre elas, as endêmicas para dirofilariose.

Comparando os portadores de dirofilariose e a eosinofilia apresentada no leucograma, houve uma forte correlação positiva entre eles. É muito comum observar esta alteração no hemograma, uma vez que a eosinofilia em cães está intimamente relacionada à presença de ectoparasitas e endoparasitas, bem como doenças de curso alérgico. Isso se deve ao fato de que o leucócito atua neutralizando a histamina e componentes de ativação do sistema complemento, gerado no local da infecção parasitária (JERICÓ et al., 2015; WALCHER et al., 2013).

Em relação as afecções respiratórias, a bronquite crônica canina foi a mais prevalente. Isso pode estar associado ao fato de a bronquite crônica ser mais comum em cães de meia idade a idosos e em raças de pequeno porte, como visto nesse estudo. Além disso, pode estar relacionado a proximidade dos cães com seus tutores, os quais os humanizam. A utilização direta de perfumes ou roupas fortemente perfumadas em cães, e os demais contatos de proximidade com seu tutor, como dormir na mesma cama, estar no ambiente em que este fuma, e até mesmo permanecer em ambientes com ar-condicionado ligado por muito tempo, são fatores que ajudam a desencadear a inflamação dos brônquios e bronquíolos (FILHO et al., 2019) e justificam o grande predomínio da afecção na rotina clínica de pequenos animais, a se pensar, principalmente, no serviço especializado.

O colapso de traqueia foi a segunda afecção respiratória mais prevalente do estudo. A literatura aponta que a cronicidade de afecções das vias aéreas e processos inflamatórios, associados a predisposição da frouxidão da musculatura dorsal traqueal são fatores potenciais desencadeantes desta doença. Diante disso, por se tratar de uma doença progressiva e degenerativa, a idade dos animais do presente estudo, além da alta incidência de cães com endocardiose e bronquite crônica, justificaria a ocorrência 
desta afecção (REINERO; MASSEUAU; 2021; TAPPIN, 2016). Outra explicação poderia seria a grande incidência de raças com tendência e predisposição anatômica ao colapso de traqueia, como Yorkshire Terrier, Poodle, Chihuahua, Shih Tzu e Lhasa Apso, relatadas nesse estudo (DELLA MAGGIORE, 2019).

Os quadros de broncopneumonia relatados no estudo, podem estar relacionados aos estágios mais avançados de diversas doenças, como o caso da bronquite crônica canina e colapso de traqueia. Doenças respiratórias crônicas propiciam a instalação de infecções secundárias, principalmente pela diminuição da imunidade do animal e pelos danos gerados nas vias aéreas. Os fatores citados anteriormente, favorecem a proliferação de microrganismos que residem na microbiota respiratória do próprio paciente, gerando o desenvolvimento da broncopneumonia (GONZALES; KING, 2018; ROZANSKI, 2014). Diante do exposto, o fato dos animais apresentarem a bronquite crônica e colapso de traqueia como achado acidental no exame pré-operatório, poderia justificar a incidência da broncopneumonia neste estudo, visto que não houve tratamento prévio nos animais até a chegada desta avaliação, contribuindo assim para a progressão da doença.

A síndrome braquicefálica também teve destaque dentre as principais doenças das vias aéreas. A grande incidência de cães da raça braquicefálica no estudo poderia explicar o achado, visto que a popularidade das raças braquicefálicas é um fator que contribui para alta prevalência da síndrome (EMMERSON, 2014; OECHTERING, 2010). Entretanto, quando comparado a incidência da doença ao número de animais de raças braquicefálicas, esta apresentou-se baixa. Por ser tratar de uma doença multifatorial e de origem congênita, espera-se que o diagnóstico ocorra em animais mais jovens, com idades entre dois e três anos (MEOLA, 2013; RIECKS et al., 2007), dado que diverge com os achados do presente estudo, o qual apresentou animais de idade avançada, justificando o pequeno número de cães com a síndrome. Ademais, a obstrução respiratória decorrente da síndrome, em longo prazo, tende a causar hipertensão pulmonar, com consequente dilatação e hipertrofia compensatória do ventrículo direito, refletindo em diminuição do débito cardíaco, gerando taquicardia sinusal (SMITH et al., 2015), achado eletrocardiográfico frequente neste estudo, para estes pacientes.

Uma possível explicação para a prevalência de arritmia sinusal nesse trabalho, pode ser a grande incidência de cães braquicefálicos sem condições clínicas de hipertensão pulmonar, visto que essa alteração está associada ao aumento da atividade parassimpática no nodo sinoatrial (BOSQUET et al., 2007), apesar de estudos, como o de Filho (2020), denotarem um indicador de redução do tônus simpático. Em relação ao ritmo sinusal e taquicardia sinusal, estes podem ter sido prevalentes em decorrência do estresse e maior ativação do sistema nervoso simpático nos cães braquicefálicos ou pacientes que em condições de normalidade teriam a arritmia sinusal, uma vez que por estarem em um ambiente diferente do seu convívio social cotidiano, não estavam acostumados com a manipulação. A justificativa citada anteriormente, pode ainda, explicar a prevalência de taquicardia sinusal, principalmente em animais de outras raças, as quais em situações de estresse, apresentam aumento da frequência cardíaca (TILLEY; BURTINICK, 2004).

A taquicardia ventricular, ritmo cardíaco iniciado nos ventrículos por pontos ectópicos, apresentou-se apenas em um animal, relacionando-se com o único caso abordado de um paciente com neoplasia esplênica. Isso se deve a liberação de citocinas tóxicas, que possuem alto tropismo pelo sistema de condução cardíaco, cursando com alterações eletrofisiológicas em nível dos cardiomiócitos, bem como o aumento de radicais livres nestes pacientes. A fim de confirmação, evidenciou-se normalização do ritmo cardíaco após a esplenectomia, e na revisão cardiológica, observou-se normalização do eletrocardiograma, confirmando que a arritmia detectada se deu por origem extra-cardíaca (ELLIOT, 2014).

Nas alterações de condução do impulso elétrico, foi constatado uma maior frequência de sinus arrest, seguido de BAV de primeiro e segundo graus. Estes três ritmos podem ser causados por terapia com algumas drogas, tônus vagal excessivo, distúrbios eletrolíticos, e aumento atrial devido insuficiência mitral (WARE, 2015). Não foi possível verificar se os bloqueios atrioventriculares encontrados em alguns cães estavam associados à farmacoterapia prévia. Diante disso, o fato de $20 \%$ dos animais em ICC apresentarem endocardiose de mitral como principal causa (SANTILLI et al., 2020), poderia justificar os achados de bloqueios, sendo ainda acrescidos quanto a pacientes com comorbidades em doenças respiratórias, que possuem alta relação na dinâmica do sistema nervoso autônomo ao que diz respeito o ciclo da inervação vagal sob o sistema de condução cardíaco, aliado aos pacientes em sobrepeso, como evidenciado por Filho 2019.

Os achados hematológicos revelam que os valores médios de hematimetria se encontram acima dos valores de referência, e com discreta variação, podendo remeter a cães em quadro de hipóxia, uma vez que este quadro estimula a produção de eritrócitos. Esta informação é reforçada quando feita a correlação entre os níveis de hemácias e cães cardiopatas, a qual demonstrou-se positiva. Devido ao baixo débito cardíaco no início da ICC, há a diminuição da perfusão em órgãos alvos, como por exemplo os rins (WARE, 2015). A diminuição do fluxo sanguíneo renal, estimula a síntese de eritropoietina, hormônio que induz a produção de eritrócito, aumentando os níveis de hemácia. Por outro lado, devido a diminuição das pressões de enchimento e volume de saída do lado esquerdo do coração na ICC, o animal acaba desenvolvendo edema pulmonar, o qual afeta a ventilação das regiões pulmonares, levando a um quadro de hipóxia (ERIKSON, 2006).

Outra correlação observada foi a dos pacientes portadores de ICC associando-se de maneira moderada com nível de proteínas plasmáticas. Esta informação pode estar relacionada a baixa perfusão encontrada na ICC. Por outro lado, pode estar associada com a síndrome cardiorrenal em pacientes já em congestão, em que há perda de proteína pela urina, favorecendo a movimentação de líquidos do espaço vascular para o interstício (BERTO et al., 2020). Ainda assim, pode ser associada a casos de hepatopatias secundária a insuficiência cardíaca, principalmente nos quadros congestivos direito, na qual o fígado reduz a produção de albumina (principal proteína plasmática), ou relacionada também a hipofunção hepática, estabelecido pelos valores de ureia sérica (NASCHITZ et al., 2000).

A média das plaquetas apresentou-se abaixo dos valores de referência. Esse dado pode estar relacionado ao fato de muitos cães serem pacientes oncológicos. Geralmente, a trombocitopenia é uma anormalidade hemostática muito 
comum em animais portadores de neoplasias. Essa alteração é resultado da produção diminuída de plaquetas pela medula óssea à liberação de citocinas inibidoras ou aumento do consumo/destruição de plaquetas por alterações endoteliais (ELLIOT, 2014).

Comparando-se a idade com a leucometria, observou-se moderada correlação negativa. Isso pode estar relacionado ao processo fisiológico de redução progressiva das funções biológicas durante o envelhecimento. De acordo com Hoskins (2008) os animais mais velhos são menos capazes de combater as infecções, devido a redução da fagocitose e quimiotaxia. Um achado importante e marcante nos pacientes tidos com alterações inflamatórias e infeciosas marcantes, como doenças periodontais e neoplasia mamária, sem responder com leucocitose para debelar estes focos.

A correlação existente, mesmo que fraca, entre o hematócrito e os níveis de creatinina, pode estar diretamente associada ao fato de a população de animais estudada ter pacientes mais susceptíveis a doença renal crônica, devido a idade mais avançada e, também, pela disfunção cardíaca apresentada e outras comorbidades. A DRC causa a diminuição da síntese de eritropoietina pelos rins, culminando, desta forma, na diminuição de células vermelhas (TORRES et al., 2017).

Em relação a ureia, foi possível observar seu valor médio dentro dos valores de referência, entretanto, o valor máximo elevado, remetendo a cães já em azotemia, principalmente aqueles com a síndrome cardiorrenal estabelecida, provavelmente influenciando para os valores máximos obtidos (LOPES; VEIGA, 2008; NICOLLE et al. 2007).

Quanto a bioquímica hepática, houve correlação entre ALT e albumina, bem como AST e FA e AST e albumina. A relação entre as enzimas de extravasamento e a albumina, pode estar relacionada diretamente ao órgão na qual indicam comprometimento, nesse caso, o fígado. A albumina por ser exclusivamente sintetizada pelo fígado, se correlaciona de maneira forte com a ALT justamente por essa ser mais hepato-

\section{Referências}

ALMEIDA, M.O. et al. Parasitismo de cães por microfilárias de Dirofilaria immitis: influência da raça, sexo e idade. Revista Brasileira de Saúde e Produção Animal, v. 2, n. 3, 2001.

ALLISON, R.W. Avaliação laboratorial da função hepática. In: THRALL, M.A., WEISER, G., ALLISON, R.W., CAMBELL, T. W. (eds). Hematologia e Bioquímica Clínica Veterinária. 2. ed. São Paulo: Rocca, 2015. p. 853-903.

ATAIDE, W.F. et al. Estudo retrospectivo dos procedimentos cirúrgicos realizados em cães e gatos em um Hospital Veterinário Universitário na Região Centro-Oeste do Brasil. Brazilian Journal of Development, v. 6, n. 6, p. 35413-35422, 2020.

BERTO, A.N. et al. Síndrome Cardiorrenal tipo II: bases fisiopatológicas e terapêuticas em cães e gatos. Medicina Veterinária (UFRPE), v. 14, n. 3, p. 162-172, 2020.

BORGARELLI, M.; BUCHANAN, J.W. Historical review, epidemiology and natural history of degenerative mitral valve disease. Journal of Veterinary Cardiology, v. 14, n. 1, p. 93-101, 2012.

BOSQUET, L.; GAMELIN, F.X.; BERTHOIN, S. A resistência aeróbia é um determinante da regulação autonômica cardíaca? Jornal europeu de fisiologia aplicada, v. 100, n. 3, pág. 363-369, 2007. específica quando comparada a AST, a qual apresenta-se em maior abundância nas células musculares. A relação moderada entre AST e FA pode estar associada ao fato de ambas apresentarem isoenzimas extra-hepáticas, tendo aumento de suas atividades devido a danos em outros tecidos tais quais tecidos ósseos, musculares e em sistema hemolinfático, o que se relaciona as principais afecções de base, como a doença periodontal, neoplasias e doenças ortopédicas (ALLISON, 2015).

\section{Conclusões}

A doença degenerativa crônica de válvula mitral, dirofilariose, bronquite crônica canina e colapso de traqueia são afecções prevalentes em cães atendidos por um setor especializado de cardiologia e doenças respiratórias, sendo as raças de pequeno porte as mais afetadas, com diferença entre os gêneros, observando maior número de fêmeas, principalmente decorrente ao tipo de procedimento cirúrgico indicado.

Pacientes cardiopatas ou com doença respiratória apresentaram alterações do ritmo e achados laboratoriais, comorbidades, como doença periodontal e doença renal crônica, além do motivo do procedimento cirúrgico como, neoplasias mamárias e doenças articulares. Isso demonstra o caráter multifatorial a ser observado no paciente no momento da avaliação pré-operatória.

Foi possível concluir que doenças cardíacas em estágios de congestão possuem redução da pressão arterial, aumento da hematimetria e taquiarritmias, como taquicardia sinusal e/ou complexos atriais prematuros. Além disso, pode-se perceber grande número de cães portadores assintomáticos de dirofilariose, os quais cursaram com aumento de eosinófilos.

Pacientes com idade mais avançada tendem a ter redução dos valores de leucometria global e maior probabilidade de manifestação de aumento da pressão arterial.

Estudos com populações maiores e com mais dados são importantes para o entendimento da epidemiologia e sua correlação com os demais achados dessas doenças em cães.

CARVALHO, C. F. et al. Eletrocardiografia pré-operatória em 474 cães. Arquivo Brasileiro de Medicina Veterinária e Zootecnia, v. 61, n. 3, p. 590-597, 2009.

CASTRO, M. G. et al. Estudo retrospectivo ecodopplercardiográfico das principais cardiopatias diagnosticadas em cães. Arquivo Brasileiro de Medicina Veterinária e Zootecnia, v. 61, p. 1238-1241, 2009.

DELLA MAGGIORE, A. Uma atualização sobre colapso da traqueia e das vias aéreas em cães. Veterinary Clinics of North America: Small Animal Practice, v. 50, n. 2, pág. 419-430, 2019.

DROBATZ, K. J. et al. Textbook of small animal emergency medicine. John Wiley \& Sons, 2018.

ELLIOTT, J. Síndromes paraneoplásicas em cães e gatos. In Pratice, v. 36, n. 9, p. 443-452, 2014.

EMMERSON, T. Brachycephalic obstructive airway syndrome: a growing problem. Journal of Small Animal Practice, v. 55, n. 11, p. 543-544, 2014.

ERIKSON, E. O Sistema Cardiovascular. In: DUKES, H.H. (ed). Fisiologia dos animais domésticos. 2 ed. Rio de Janeiro: Guanabara Koogan, 2006. p. 163-329. 
FILHO, M. S. et al.. Canine heartworm: natural infection along remote coastal area of Rio de Janeiro. Brazilian Journal of Veterinary Medicine, v. 43, n. 1, p. e000220-e000220, 2021.

FILHO, M. S. et al. Bronquite crônica canina-revisão de literatura. Medicina Veterinária (UFRPE), v. 13, n. 3, p. 329-337, 2019

FILHO, M. S. et al.. Study of the heart rate variability in dogs with brachycephalic syndrome submitted to rhinoplasty surgery. Brazilian Journal of Veterinary Medicine, v. 42, n. 1, p. e104919-e104919, 2020

GONZALEZ, A.L.; King, L.G. Broncopneumonia. In: DROBATZ, K.J., HOPPER, K., ROZANSKI, E.A.; SILVERSTEIN, D.C. (ed.). Textbook of Small Animal Emergency Medicine. John Wiley \& Sons. 2018. p. 234-241.

HAGGSTROM, J.; KYART, C.; PEDERSEN, H. D. Acquired valvular heart disease. In: ETTINGEER, S.J.; FELDMAN, E.C. (ed.) Textbook of veterinary internal medicine. 5 ed. Filadélfia: WB Saunders. 2010

HOSKINS, J.D. Geriatria e Gerontologia do cão e gato. São Paulo: Roca, 2008. 234p.

JAIN, N.C. Essentials of veterinary hematology. Philadelphia: Lea \& Febiger, 1993.

JERICÓ, M.M.; NETO, J.P.A.; KOGIKA, M.M. (2015). Tratado de Medicina Interna de cães e gatos. In: Gomes, R. G. S. Hematologia e Doenças Imunomediadas. Rio de Janeiro: Roca, 2015. v. 2, cap. 203, p. 1850-1851.

KANEKO, J.J., HARVEY, J.W., BRUSS, M.L. Clinical biochemistry of domestic animals. New York: Academic Press, 1997.

KEENE, B.W. et al. ACVIM consensus guidelines for the diagnosis and treatment of myxomatous mitral valve disease in dogs. Journal of veterinary internal medicine, v. 33, n. 3, p. 11271140, 2019.

KITTLESON, M.D. Therapy of Heart Failure. In: ETTINGER, S.J., FELDMAN, E.C. (eds). Textbook of Veterinary Internal Medicine. St. Louis, Missouri, USA. Saunders, 2005. p. 713-736.

DALMAS, E.G. O comportamento do consumidor de produtos e serviços do mercado pet quanto aos cuidados com os animais de estimação. (p. 69). Trabalho de Conclusão de Curso (Bacharel em Administração) - Universidade de Caxias do Sul, Bento Gonçalves. 2019.

LOPES, S.T.A.; VEIGA, A. Urinálise. In: Gonzáles, F. H. D., \& Silva S. C. (eds). Patologia Clínica Veterinária: Texto Introdutório. Porto Alegre: Universidade Federal do Rio Grande do Sul, p. 107139, 2008.

MEOLA, S.D. Brachycephalic airway syndrome. Topics in Companion Animal Medicine, v. 28, n. 3, p. 91-96, 2013.

MEUTEN, D. Avaliação e Interpretação Laboratorial do Sistema Urinário. In: Thrall, M. A., Weiser, G., Allison, R. W., \& Cambell, T. W. (eds). Hematologia e Bioquímica Clínica Veterinária. São Paulo: Rocca, p. 689-806, 2015.

METZGER, F. L. Senior and geriatric care programs for veterinarians. The Veterinary clinics of North America. Small animal practice, v. 35, n. 3, p. 743-753, 2005.
NASCHITZ, J.E., et al. Heart diseases affecting the liver and liver diseases affecting the heart. American heart journal, v. 140, n. 1, p. 111-120, 2000.

NICOLLE, A.P., et al. Azotemia and glomerular filtration rate in dogs with chronic valvular disease. Journal of veterinary internal medicine, v. 21, n. 5, p. 943-949, 2007.

OECHTERING, G. Síndrome braquicefálica: novas informações sobre uma antiga doença congênita. Veterinary Focus, v. 20, n. 2, p. 10-18, 2010.

RASMUSSEN, C.E. et al. Heart rate, heart rate variability, and arrhythmias in dogs with myxomatous mitral valve disease. Journal of veterinary internal medicine, v. 26, n. 1, p. 76-84, 2012.

REINERO, C. R.; MASSEAU, I. Lower airway collapse: Revisiting the definition and clinicopathologic features of canine bronchomalacia. The Veterinary Journal, p. 105682, 2021.

ROCHA, M.L. et al. Qualidade de vida de cães e gatos idosos. PUBVET, v. 7, p. 259-311, 2013.

ROZANSKI, E. Canine chronic bronchitis. Veterinary Clinics: Small Animal Practice, v. 44, n. 1, p. 107-116, 2014.

RIECKS, T.W., BIRCHARD, S.J.; STEPHENS, J.A. Surgical correction of brachycephalic syndrome in dogs: 62 cases (19912004). Journal of the American Veterinary Medical Association, v. 230, n. 9, p. 1324-1328, 2007.

SANTILLI, R., et al. Eletrocardiografia de cães e gatos: Diagnóstico de arritmias. São Paulo: Editora MedVet, 2020.

SANTOS, W.F., et al. Prevalência da doença Valvar Degenerativa Crônica de Mitral e da bronquite crônica diagnosticadas em avaliação pré-operatória em cães (canis familiaris) atendidos no hospital veterinário da Universidade Federal Rural do Rio de Janeiro no período de março. Revista de Educação Continuada em Medicina Veterinária e Zootecnia do CRMV-SP, v. 11, n. 2, p. 87-87, 2013.

SMITH, F.W.K., et al. Manual of canine and feline cardiology-EBook. Elsevier Health Sciences, 2015.

TAPPIN, S. W. Canine tracheal collapse. Journal of Small Animal Practice, v. 57, n. 1, p. 9-17.

TILLEY, L.P.; BURTNICK, N. L. Eletrocardiografia para o clínico de pequenos animais. São Paulo: Roca, 2004.

TORRES, M.M., et al. Relation between anaemia and bone marrow features and serum erythropoietin in dogs with chronic kidney disease. Pesquisa Veterinária Brasileira, v. 37, n. 6, p. 598-602, 2017.

WALCHER, D.L.; PEDROSO, D.; FRIZZO, M.N. Associação entre parasitoses intestinais e alterações do hemograma. Revista Mirante, v. 3, n. 1, p. 18-40, 2013.

WARE, W. A. Doença valvar endocárdica adquirida. In: NELSON, R.W.; COUTO, C.G. (eds.). Medicina interna de pequenos Animais. Rio de Janeiro, Brasil.: Elservier, 2015. p. 113-126 